BULLETIN OF THE

AMERICAN MATHEMATICAL SOCIETY

Volume 81, Number 1, January 1975

\title{
SOME DIOPHANTINE EQUATIONS RELATED TO THE QUADRATIC FORM $a x^{2}+b y^{2}$
}

\author{
BY EDWARD A. BENDER AND NORMAN P. HERZBERG
}

Communicated by Robert Fossom, September 10, 1974

Introduction. We have been preparing a survey paper on diophantine equations associated with the positive definite quadratic form $a x^{2}+b y^{2}$. The effective results of Baker [2] lead to very large explicit bounds on the number and size of solutions for the two equations discussed below. By other methods which rely heavily on the forms of the equations, these general bounds can be markedly improved. Since the general results are too lengthy to report here, we will give some particular results of special interest.

The EQuATION $a x^{2}+D=N^{z}$. Suppose $a, N$ and $D$ are positive integers, and that $N$ is prime to $2 D$. What is the number $\sigma$ of positive integral solutions $x, z$ to the equation $a x^{2}+D=N^{z}$ ? If $D$ is not an odd square and $N>D+12$, then $\sigma$ does not exceed twice the number of divisors of $h(\sqrt{-a D)}$, the class number of $Q(\sqrt{-a D)}$. We have constructed a table of bounds on $\sigma$ for $a D<25$ and believe the fact that $\sigma=1$ for the following cases of $a, D$ to be new:

$$
\begin{aligned}
(a, D)=(1,6),(2,3),(1,8),(1,10),(1,14),(1,15) \\
(5,3),(1,18),(1,20),(3,7),(1,23),(1,24) .
\end{aligned}
$$

Among the many earlier results with $\sigma=1$ we mention [5], [6]. We have also shown that when $D<4 N$ is not an odd square and there are no solutions with $z$ dividing $3 h(\sqrt{-a D)}$, then $\sigma=0$.

Because of its interest elsewhere [4], we note that $3 x^{2}+8=N^{z}$ has a solution with $z=1$ if it has any solutions, has at most two solutions, and has no second if $11<N<2.7 \times 10^{19}$.

The EQUATION $a x^{2}+D=z^{n}$. Suppose $a, n$ and $D$ are positive integers. What are the positive integer solutions of $a x^{2}+D=z^{n}$ ? If $n$ does

AMS (MOS) subject classifications (1970). Primary 10B15, 10B25, $10 \mathrm{B05}$. 
not divide $3 h(\sqrt{-a D)}$ and $D$ is not an odd square, there are no solutions with $z$ prime to $2 D$ and $z>D\left(5+(n+1)^{2} / \pi^{2}\right)$.

Suppose $D$ is square-free, $5 \nmid h(\sqrt{-a D})$, and $a D \neq 7$ modulo 8 . The equation

$$
a x^{2}+D=z^{5}
$$

with $a$ fixed has solutions for at most four values of $D$. These values of $D$ and $x$ are found [3] by solving

$$
\left(D-5 x^{2}\right)^{2}=20 a^{2} x^{4}+1 .
$$

Blass [3] showed that when $a=1$, (1) has only the two solutions $(D, z)=$ $(19,55)$ and $(341,377)$. When $a=2$, the only solution is $(D, z)=(19,21)$. If the condition " $D$ square-free" is replaced by " $D$ free of fifth powers and $D<4 z$," the solutions of (1) are still determined by (2).

SKETCH OF PROOFS. The proofs are based on an analysis of the solutions $z, x(z), y(z)$ of the equation $a x(z)^{2}+b y(z)^{2}=N^{z}$ where $a b$ is square-free. To avoid complications here, assume that $a>1, b>1$, and that $N$ is an odd prime. Standard arguments over the algebraic number field $Q(\sqrt{-a b})$ show that all solutions are given by

$$
a x(r l) \pm \sqrt{-a b} y(r l)=a^{(r-1) / 2}(a x(l)+\sqrt{-a b} y(l))^{r}
$$

where $r$ is odd and the smallest solution is at $z=l$. A $q$-adic argument [1] and congruential arguments based on (3) restrict the number of solutions with fixed $y$. An Argand diagram argument provides a lower bound on $r$ when $y(r l)$ is small.

\section{REFERENCES}

1. R. Apéry, Sur une équation diophantienne, C. R. Acad. Sci. Paris 251 (1960), 1451-1452. MR 22,\#10950

2. A. Baker, Bounds for the solutions of the hyperelliptic equation, Proc. Cambridge Philos. Soc. 65 (1969), 439-444. MR 38 \#3226.

3. J. Blass, On the diophantine equation $Y^{2}+K=X^{5}$, Bull. Amer. Math. Soc. 80 (1974), 329.

4. N. Herzberg, Integer solutions of $b y^{2}+p^{n}=x^{3}, \mathrm{~J}$. Number Theory (to appear).

5. W. Ljunggren, On the diophantine equation $C x^{2}+D=y^{n}$, Pacific J. Math. 14 (1964), 585-596. MR 28 \#5035.

6. T. Nagell, Contributions to the theory of a category of diophantine equations of the second degree with two unknowns, Nova Acta Soc. Sci. Upsal. (4) 16 (1955), no. 2, 38 pp. MR 17, 13.

INSTITUTE FOR DEFENSE ANALYSES, 100 PROSPECT AVENUE, PRINCETON, NEW JERSEY 08540

Current address (E. A. Bender): Department of Mathematics, University of California at San Diego, La Jolla, California 92037 